\title{
Constructive effects of diversity in a multi-neuron model of the homeostatic regulation of the sleep-wake cycle
}

\author{
Marco Patriarca ${ }^{\mathrm{a}}$, Emilio Hernández-García ${ }^{\mathrm{b}}$, Raúl Toral ${ }^{\mathrm{b}}$ \\ ${ }^{a}$ NICPB-National Institute of Chemical Physics and Biophysics, Rävala 10, 15042 \\ Tallinn, Estonia \\ ${ }^{b}$ IFISC-Instituto de Física Interdisciplinar y Sistemas Complejos (CSIC-UIB), E-07122 \\ Palma de Mallorca, Spain
}

\begin{abstract}
As an instance of diversity-induced resonance and of the constructive role of heterogeneity in complex systems, here we study a generalized version of a physiologically-motivated sleep-wake cycle model taking into account the role of orexin [M. Patriarca, E. Hernandez-Garcia, R. Toral, S. Postnova, H. A. Braun, PLoS Comput. Biol. 8 (2012) e1002650; S. Postnova, K. Voigt H. A. Braun, J. Biol. Rhythms 24 (2009) 523]. With respect to the versions of the model studied previously, here we consider systems containing both a set of orexin neurons - responsible for the production of orexin - and a set of glutaminergic neurons - representing mental activity or sleep depending on their firing or silent state, respectively — within some basic network topologies. The neurons of one or both types are diversified and it is shown how the interplay between the heterogeneous constituent units produces as an emergent effect the recovering or improving of the sleep-wake cycle. At a general level, the results obtained suggest that also systems with a dynamics driven by a homeostatic mechanism, with a time scale much longer than that of the constituent excitable units, may present diversity-induced resonance.
\end{abstract}

Keywords:

Emergent phenomenon; Complex System; Diversity-Induced Resonance; Neuronal Dynamics; Wake-Sleep Cycle;

Email addresses: marco.patriarca@kbfi.ee (Marco Patriarca), emilio@ifisc.uib-csic.es (Emilio Hernández-García), raul@ifisc.uib-csic.es (Raúl Toral) 


\section{Introduction}

In this paper we study a multi-neuronal model aimed at modelling at a microscopic physiological level the homeostatic dynamics of the sleep-wake cycle observed in many species. The multi-neuron nature of the system under study is sufficient to consider it a classical example of complex systems, since the corresponding model is obtained by assembling a set of excitable units (Hodgkin-Huxley neurons) interacting with each other nonlinearly. Systems of this kind are known to produce emergence of new properties and selforganized behaviors.

However, the type of self-organization searched in the system under study concerns an additional aspect that only recently has been recognized as a candidate to be another universal feature of many complex systems, namely the heterogeneity of the constituents units. As shown below, one can produce a better and more stable periodicity of the wake-sleep cycle induced by a homeostatic dynamics in a set of heterogeneous neurons than in one of homogeneous ones, if the level of heterogeneity is neither too low nor too high. Namely, the model considered below presents evidence of diversityinduced resonance, an emergent effect originally discovered within a mechanical model, analogous to those producing stochastic resonance, but where the heterogeneity of the units replaces the level of noise in producing an optimal response to an external signal for a suitable intermediate degree of heterogeneity $[1,2,3]$. Diversity-induced resonance has been by now found in different systems with different features and dynamical time and space scales $[4,5,6,7,8]$.

Here we consider the question whether diversity-induced resonant effects, already found in the encoding response of neuronal assemblies [8], have a relevant role also when affecting neurons undergoing a homeostatic regulation with the time scale of the circadian clock. Namely, we study the effects of diversity in a physiological model of the sleep-wake cycle, based on the two-neuron model introduced in Ref. [9] depicted in Fig. 1. The original model is minimal in that it consists of one orexin-producing neuron and one glutaminergic neuron, working according to a homeostatic mechanism for the regulation of sleep based on depression of orexinergic synapses during wakefulness and their recovery during sleep. The structure of the model reflects experimental findings about the role of the orexin system in maintaining wakefulness and its ability to integrate sleep-wake relevant information from many brain areas $[10,11]$ and respond to changes in the body external and 
internal environments by encoding the body activity state, energy balance, sensory and emotional stimuli $[12,13]$. In that model the orexin neuron $\mathrm{A}$

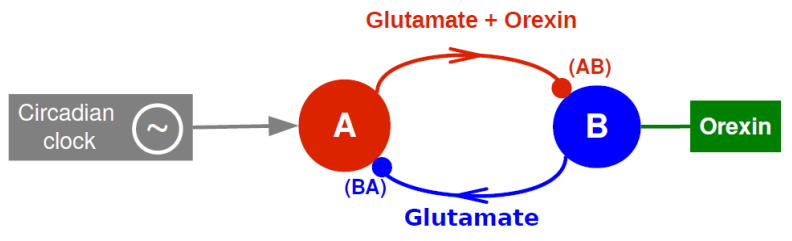

Figure 1: Two-neuron model with one orexin-producing neuron (A) acted upon by a circadian signal represented by the gray box and one glutaminergic neuron (B) undergoing the homeostatic effects of Orexin represented by the green box. The two neurons can interact with each other through the Orexin and the glutamate channels. See Ref. [9] and the text for further details.

and the glutaminergic neuron B are reciprocally connected to each other according to the experimentally established physiological connections [14], see Fig. 1. Both the orexin and the glutamate neuron are firing during wakefulness and are silent during sleep. The transitions between firing and silence are governed by the interplay between the circadian input and homeostatic mechanisms as initially proposed by Borbely [15]. Only one type of orexin neurotransmitter is considered and it is assumed that the system can be either in the wake state or in a generic (non-Rapid Eye Movement) sleep state.

Motivated by the constructive role of disorder discussed above and the well-known fact that neurons are highly heterogeneous by their nature $[10$, $18,13]$, we have started studying the effects of diversity in a previous work, where we have analyzed the role of heterogeneity in the neuronal A-B couplings within the framework of a heterogeneous generalization of the model of Ref. [9]. In such a model the orexin-producing A-neuron was replaced with a set of A-neurons connected in parallel to the B-neuron (the B-neuron feels the average of the corresponding interactions), see the scheme in Fig. 2. The model presents evidence of diversity-induced resonance $[16,17]$. It was found that the most robust sleep-wake cycle, as defined according to a suitable sleep quantifier (see Sec. 3 below), corresponds to an intermediate level of diversity in the mutual dynamical couplings between neurons.

As a further step toward a more realistic physiological modelling of the sleep-wake dynamics, here we extend further the models we have studied previously considering model systems where there is both an assembly of 


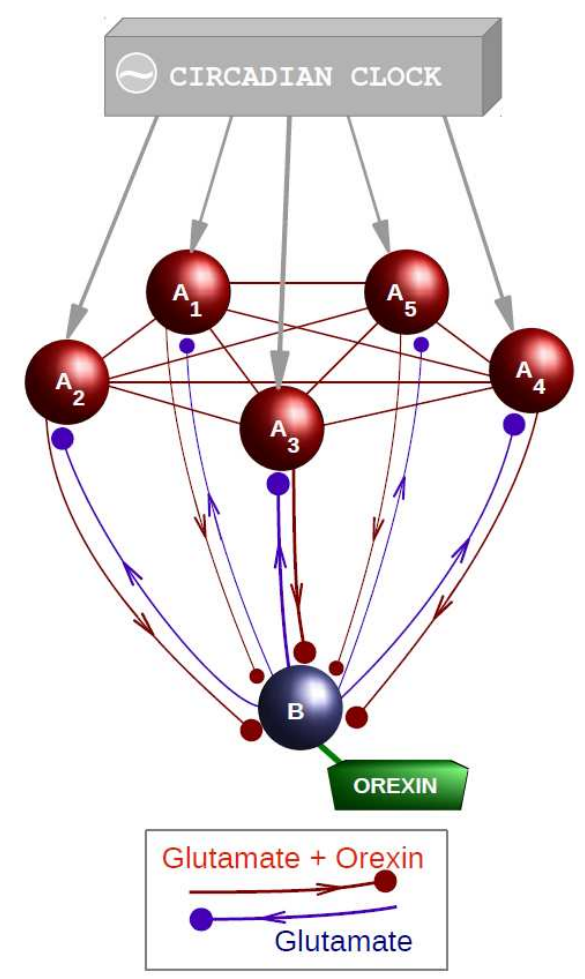

Figure 2: Example of heterogeneous model of Refs. [16, 17], in which the orexin-producing A-neurons, acted upon by a circadian signal (gray box), interact in parallel with the (same) glutaminergic B-neuron, that undergoes the homeostatic effects induced by Orexin (represented by the green box). See text for details.

$N_{\mathrm{A}}$ orexin-producing neurons (A-neurons in the following) and an assembly of $N_{\mathrm{B}}$ glutaminergic neurons (B-neurons), where in principle all neurons of one set are not perfectly identical to each other. In this exploratory study, we consider below some simple topologies for the local networks of A- and B-neurons and the coupling between them.

\section{The model}

The dynamics of the model studied is based on Hodgkin-Huxley-type neurons, as in the two-neuron model and in its generalization with heterogeneous orexin-producing neurons $[9,16,17]$. The model set up is represented in Fig. 3. The system contains a set $\mathbb{A}$ of $N_{\mathrm{A}}$ orexin neurons acted upon 


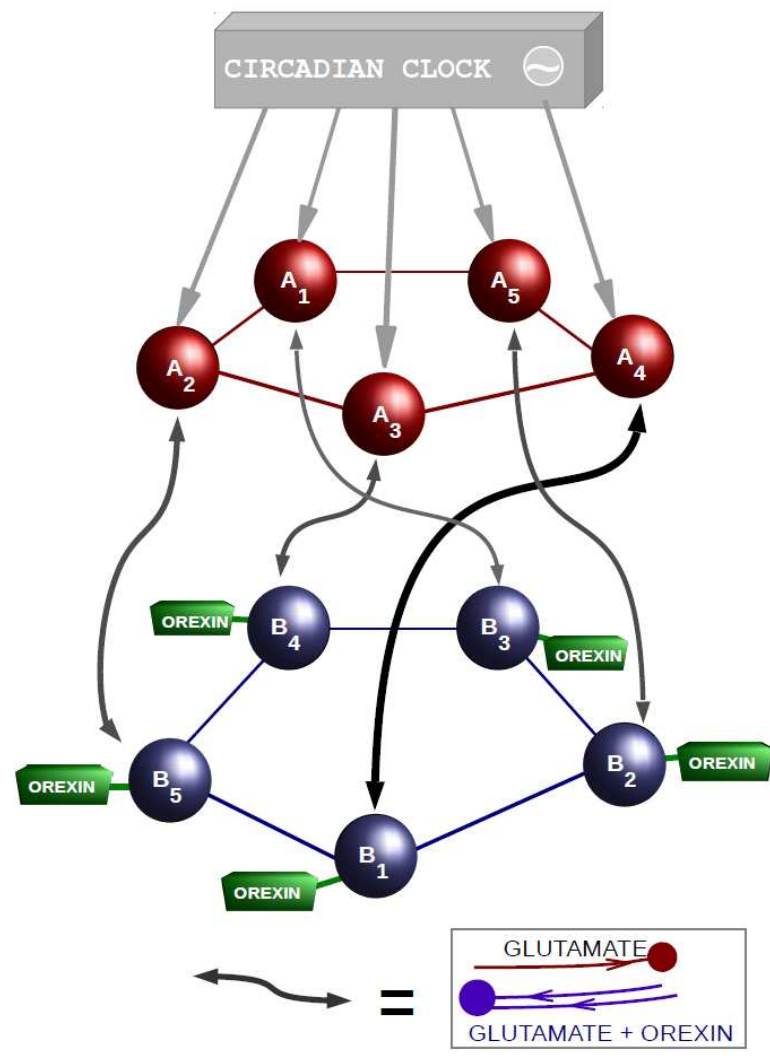

Figure 3: Example of the heterogeneous model considered in the present paper, with $N_{\mathrm{A}}=5$ Orexin-producing A-neurons and $N_{\mathrm{B}}=5$ glutaminergic B-neurons, in which neurons of the same type form a local ring-network and interact through a simple $V-V$ current. Each A-neuron interacts with a B-neuron as in the original model of Ref. [9], each B-neuron undergoing a homeostatic regulation (represented by the respective green box) induced by Orexin.

by a signal in pace with the circadian rhythm, interacting with a set $\mathbb{B}$ of $N_{\text {B }}$ glutaminergic neurons. All neurons could in principle interact with each other but in the next section only a few standard configurations are considered. The interaction configuration is conveniently represented as a network in which nodes represent neurons and links their mutual interactions, so that it is natural to introduce the corresponding directed adjacency matrix $\mathcal{K}=\left\{\mathcal{K}_{m, n}\right\}$ containing the information on the presence $\left(\mathcal{K}_{m, n}=1\right)$ or absence $\left(\mathcal{K}_{m, n}=0\right)$ of a projection from neuron $m$ to neuron $n$. The type of 
interaction explicitly depends on the types $m$ and $n$ of the two neurons connected: if $m \in \mathbb{A}$ and $n \in \mathbb{B}$, there is a glutamate and an orexin projection from $m$ to $n$ and only a glutamate projection from $n$ to $m$; if both neurons $m$ and $n$ belong to the same population, either $\mathbb{A}$ or $\mathbb{B}$, then they interact through a current linear in the potential difference (see below). For the sake of clarity we explicitly divide the total current of a neuron into the contributions coming from interactions with neurons of the same type and those due to interactions with neurons of the other type. To this aim it is convenient to use index $i$ (and $i^{\prime}$ ) for A-neurons only and to reserve index $j$ (and $j^{\prime}$ ) for B-neurons. Then the dynamical equations for the membrane potential of the generic $i$-th A-neuron $\left(i=1, \ldots, N_{\mathrm{A}}\right)$ and $j$-th B-neuron $\left(j=1, \ldots, N_{\mathrm{B}}\right)$ read

$$
\begin{aligned}
C_{\mathrm{A}} \frac{d V_{\mathrm{A}}^{i}}{d t} & =-I_{\mathrm{A}, \mathrm{L}}^{i}-I_{\mathrm{A}, \mathrm{Na}}^{i}-I_{\mathrm{A}, \mathrm{K}}^{i}-\sum_{i^{\prime} \in \mathbb{A}} \mathcal{K}_{i, i^{\prime}} I_{\mathrm{A}, \text { local }}^{i, i^{\prime}}-\sum_{j \in \mathbb{B}} \mathcal{K}_{i, j} I_{\mathrm{A}, \mathrm{gl}}^{i, j}+I_{\mathrm{ext}}(t),(1) \\
C_{\mathrm{B}} \frac{d V_{\mathrm{B}}^{j}}{d t} & =-I_{\mathrm{B}, \mathrm{L}}^{j}-I_{\mathrm{B}, \mathrm{Na}}^{j}-I_{\mathrm{B}, \mathrm{K}}^{j}-\sum_{j^{\prime} \in \mathbb{B}} \mathcal{K}_{j, j^{\prime}} I_{\mathrm{B}, \text { local }}^{j, j^{\prime}}-\sum_{i \in \mathbb{A}} \mathcal{K}_{j, i}\left(I_{\mathrm{B}, \mathrm{gl}}^{j, i}+I_{\mathrm{B}, \mathrm{ox}}^{j, i}\right),(2)
\end{aligned}
$$

where $C_{\mathrm{A}}$ and $C_{\mathrm{B}}$ represent the membrane capacitances per unit area. The system dynamics has to be completed with the explicit expressions of the current terms, that are given below.

On the right-hand side, for each neuron, there are - the three first usual terms associated to the internal dynamics, corresponding to the leakage (L), sodium $(\mathrm{Na})$, and potassium $(\mathrm{K})$ currents. These currents only depend on the potentials of the same neuron. The leakage current of the (either A or B) $k$ th neuron has a simple linear dependence on the neuron potential,

$$
I_{p, \mathrm{~L}}^{k}=g_{p, \mathrm{~L}}\left(V_{p}^{k}-E_{p, \mathrm{~L}}\right), \quad p=\mathrm{A}, \mathrm{B},
$$

where $k$ is the neuron label, $g_{p, \mathrm{~L}}$ the conductance, and $E_{p, \mathrm{~L}}$ the equilibrium potential. The other internal currents $I_{p, \alpha}^{k}$ with $p=\mathrm{A}, \mathrm{B}$ and $\alpha=\mathrm{Na}, \mathrm{K}$ also depend on the activation variable $a_{p, \alpha}$,

$$
I_{p, \alpha}^{k}=g_{p, \alpha}\left(V_{p}^{k}-E_{p, \alpha}\right) a_{p, \alpha}^{k}, \quad \alpha=\mathrm{Na}, \mathrm{K}, \quad p=\mathrm{A}, \mathrm{B},
$$

where $k, g_{p . \alpha}$, and $E_{p, \alpha}$ have analogous meanings. The time evolution of the activation variables $a_{p, \mathrm{Na}}$ and $a_{p, \mathrm{~K}}$ depends in turn on the membrane potential $V_{p}^{k}$ of the same neuron $p$,

$$
\frac{d a_{p, \alpha}^{k}}{d t}=-\frac{1}{\tau_{p, \alpha}}\left[a_{p, \alpha}^{k}-\Phi_{p, \alpha}\left(V_{p}^{k}\right)\right], \quad \alpha=\mathrm{Na}, \mathrm{K}, \quad p=\mathrm{A}, \mathrm{B},
$$


where $\tau_{p, \alpha}$ is the relaxation time scale. The equilibrium value of the activation variable is determined by a sigmoid function (also used for the other activation variables considered below)

$$
\Phi_{p, \alpha}(V)=\frac{1}{1+\exp \left[-S_{p, \alpha}\left(V-W_{p, \alpha}\right)\right]},
$$

with the two parameters given by the threshold $W_{p, \alpha}$ and slope $S_{p, \alpha}$. However, for the faster relaxation of the $\mathrm{Na}$ current it is further assumed that the relaxation time $\tau_{\mathrm{A}, \mathrm{Na}}$ is very small so that the corresponding activation variable is instantaneously relaxed, replacing the solution of Eq. (5) by its steady-state value, i.e. $a_{p, \mathrm{Na}}^{k} \equiv \Phi_{p, \mathrm{Na}}\left(V_{p}^{k}\right)$.

The following current terms on the right hand side, $I_{\mathrm{A}, \text { local }}^{i, i^{\prime}}$ and $I_{\mathrm{B}, \text { local }}^{j, j^{\prime}}$, describe the A-A or B-B interactions between neurons of the same type. They depend on the couplings represented by the corresponding elements of the adjacency matrix $\mathcal{K}$. For the sake of simplicity they are assumed proportional to the difference of their membrane potentials,

$$
\begin{aligned}
& I_{\mathrm{A}, \text { local }}^{i, i^{\prime}}=\kappa_{\mathrm{A}}\left(V_{\mathrm{A}}^{i}-V_{\mathrm{A}}^{i^{\prime}}\right), \\
& I_{\mathrm{B}, \text { local }}^{j, j^{\prime}}=\kappa_{\mathrm{B}}\left(V_{\mathrm{B}}^{j}-V_{\mathrm{B}}^{j^{\prime}}\right),
\end{aligned}
$$

where $\kappa_{\mathrm{A}}$ represents the coupling constant (conductivity) and $i, i^{\prime}$ the corresponding neuron labels of the $\mathbb{A}$ set while $\kappa_{\mathrm{B}}$ and the indexes $j, j^{\prime}$ are the analogous quantities for the $\mathbb{B}$ set.

The currents $I_{\mathrm{A}, \mathrm{gl}}^{i, j}$ and $I_{\mathrm{B}, \mathrm{gl}}^{j, i}$ describe A-B or B-A glutamate-mediated interactions between neurons of different types and are of a form similar to the currents in Eq. (4) but the dynamics of the activation variable is now determined by the potential of the neuron of the other type, i.e.

$$
\begin{aligned}
& I_{\mathrm{A}, \mathrm{gl}}^{i, j}=g_{\mathrm{A}, \mathrm{gl}}\left(V_{\mathrm{A}}^{i}-E_{\mathrm{A}, \mathrm{gl}}\right) a_{\mathrm{A}, \mathrm{gl}}^{i, j}, \\
& I_{\mathrm{B}, \mathrm{gl}}^{j, i}=g_{\mathrm{B}, \mathrm{gl}}\left(V_{\mathrm{B}}^{j}-E_{\mathrm{B}, \mathrm{gl}}\right) a_{\mathrm{B}, \mathrm{gl}}^{j, i},
\end{aligned}
$$

where the explicit equations for the activation variables for the glutamate interaction between the $i$ th A-neuron and the $j$ th B-neuron read

$$
\begin{aligned}
& \frac{d a_{\mathrm{A}, \mathrm{gl}}^{i, j}}{d t}=-\frac{1}{\tau_{\mathrm{A}, \mathrm{gl}}}\left[a_{\mathrm{A}, \mathrm{gl}}^{i, j}-\Phi_{\mathrm{A}, \mathrm{gl}}\left(V_{\mathrm{B}}^{j}\right)\right], \\
& \frac{d a_{\mathrm{B}, \mathrm{gl}}^{j, i}}{d t}=-\frac{1}{\tau_{\mathrm{B}, \mathrm{gl}}}\left[a_{\mathrm{B}, \mathrm{gl}}^{j, i}-\Phi_{\mathrm{B}, \mathrm{gl}}\left(V_{\mathrm{A}}^{i}\right)\right],
\end{aligned}
$$


and the sigmoid function $\Phi_{p, \alpha}$ is defined in Eq. (6).

Also the orexin currents $I_{\mathrm{B}, \mathrm{ox}}^{j, i}$, representing the orexin-mediated interactions between an A- and a B-neuron, are assumed to be of the form of Eq. (9),

$$
I_{\mathrm{B}, \mathrm{ox}}^{j, i}=g_{\mathrm{B}, \mathrm{ox}}\left(V_{\mathrm{B}}^{j}-E_{\mathrm{B}, \mathrm{ox}}\right) a_{\mathrm{B}, \mathrm{ox}}^{j, i} .
$$

The orexin-related part of the model contains the homeostatic mechanism, defined in the dynamics of the activation variable $a_{\mathrm{B}, \mathrm{ox}}^{j, i}$, evolving with time according to

$$
\frac{d a_{\mathrm{B}, \mathrm{ox}}^{j, i}}{d t}=-\frac{1}{\tau_{\mathrm{B}, \mathrm{ox}}}\left[a_{\mathrm{B}, \mathrm{ox}}^{j, i}-M_{i} \Phi_{\mathrm{B}, \mathrm{ox}}\left(V_{\mathrm{A}}^{i}\right)\right] .
$$

With respect to the other activation variables, this equation contains the variable $M_{i}(t) \in(0,1)$, representing the fraction of reservoir of orexin (produced by the $i$ th A-neuron) and available for neuron B: $M_{i}=0$ corresponds to an empty reservoir while $M_{i}=1$ to the maximal amount available. The last term in Eq. (12) implies that the activation of the orexin current is a combined effect of (a) the activity of the A-neuron (through the potential $V_{\mathrm{A}}^{i}$ ) and (b) a sufficient amount of orexin available to neuron B (through $M_{i}$ ). For the latter reason in Fig. 3 (as well as in the other models of Figs. 1 and 2) the homeostatic regulation induced in neurons B by the (slow) dynamics of the reservoir variables $M_{i}$ is represented symbolically by the Orexin reservoirs (green boxes) interacting with the respective B neurons.

The dynamics of the reservoir variables $M_{i}$ is the part of the model where the daily time scales $\tau_{\text {ox }}^{ \pm}$enter the problem (besides the daily period $\tau$ in the external current $\left.I_{\text {ext }}(t)\right)$,

$$
\frac{d M_{i}}{d t}=-\frac{1}{\tau_{\mathrm{ox}}^{+}}\left(M_{i}-1\right)-\frac{1}{\tau_{\mathrm{ox}}^{-}} M_{i} \Phi_{\mathrm{B}, \mathrm{ox}}\left(V_{\mathrm{A}}^{i}\right) .
$$

The first term on the right hand side of the equation is always positive and is responsible for the production of orexin while the second term, which is always negative, describes orexin consumption. The orexin-dependent part of the dynamical equations is the key-element of the model, as long as the regulation of the wake-sleep cycle is concerned, so that it is expected to be able to direct the homeostatic sleep process as follows:

(1) By initiating the wakeful part of a daily period after a sufficiently strong initial stimulus associated to the circadian rhythm that would in turn activate the B-neurons. 
(2) By maintaining wakefulness for a long time interval also after the pulse is finished, with both neurons $\mathrm{A}$ and $\mathrm{B}$ firing due to the reciprocal excitation.

(3) Re-initiating sleep by stopping the firing activity in both A- and Bneurons when the available orexin has decreased under a critical level.

The external current $I_{\text {ext }}$ represents here the mentioned daily initial stimulus, acting directly on the hypothalamus. It is assumed to be a simple rectangular pulse with height $I_{0}>0$ and a length much smaller than the daily period, $\tau_{0} \ll \tau$, which is repeated periodically at the beginning of each day, $n=0,1, \ldots$,

$$
\begin{aligned}
I_{\text {ext }}(t) & =I_{0}, \text { for } n \tau<t<n \tau+\tau_{0}, \\
& =0, \text { for } n \tau+\tau_{0}<t<(n+1) \tau,
\end{aligned}
$$

that triggers the wake up process, see also Fig. 4. In the numerical simulations the daily period $\tau$ and the other related time scales $\tau_{\text {ox }}^{ \pm}$— but not any other time scale of the problem - are rescaled for numerical convenience, in order to make the study of the system during a whole day or several days possible. The daily period has been rescaled by a factor 3600 , so that it lasts $\tau=24 \mathrm{~s}$ instead of 24 hours. This rescaling does not invalidate any of the results and conclusions, since even after the rescaled time $\tau=3600 \mathrm{~s}$ the system has reached a stable firing or silent state - depending on the parameters used, due to the fact that all the other time scales characterizing the dynamics of neurons are still much smaller than $\tau$.

The values of the model parameters, apart from the capacitances which are assumed as $C_{\mathrm{A}}=C_{\mathrm{B}}=1 \mu \mathrm{F} / \mathrm{cm}^{2}$, are listed in Table 1 .

\section{Quantifying the quality of sleep}

In order to analyze results it is necessary to introduce a quantifier of the quality of a sleep wake cycle. We first divide a day into consecutive optimal wakefulness and sleep periods of respective duration $\tau_{1}=2 \tau / 3$ and $\tau_{2}=\tau / 3$. We then introduce the quantity

$$
r=\frac{\left\langle\Delta t^{(1)}\right\rangle}{\tau_{1}}-\frac{\left\langle\Delta t^{(2)}\right\rangle}{\tau_{2}} .
$$

Here $\left\langle\Delta t^{(k)}\right\rangle, k=1,2$, is the average (over $N_{\text {sp }}$ days) of the measured wakefulness time intervals occurring during the aforementioned optimal wakefulness 


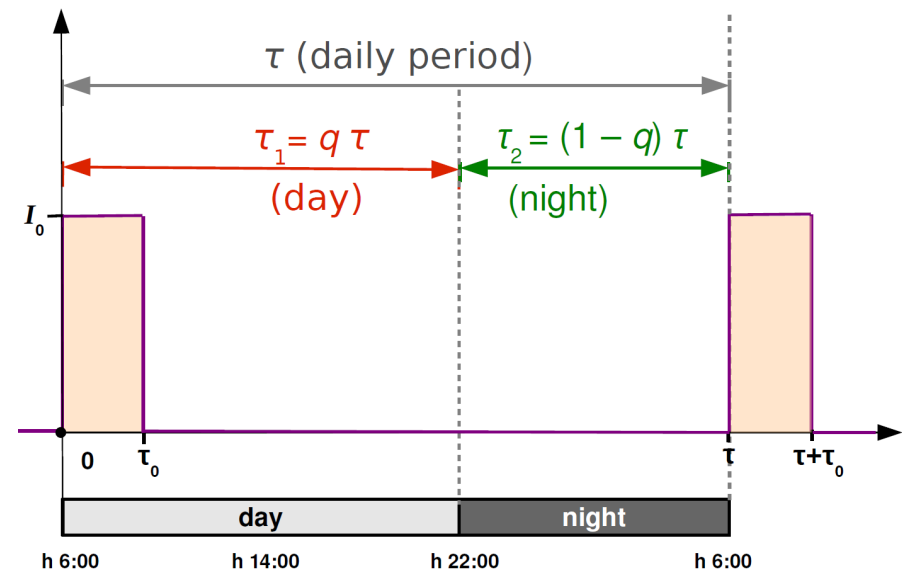

Figure 4: Two rectangular pulses of height $I_{0}$ and length $\tau_{0}$ of the external current $I_{\text {ext }}(t)$ with daily period $\tau$. Also the "ideal sleep-wake cycle", divided into a wakefulness fraction $q=2 / 3$ and a sleep fraction $1-q=1 / 3$, is shown. For clarity the sleep-wake cycle is here represented in hours, but in the numerical simulations it was convenient to rescale the daily period $\tau$ by a factor 3600, it lasts $\tau=24 \mathrm{~s}$ instead of 24 hours. See text for details.

Table 1: Parameters of the homogeneous system for the neuronal populations $p=\mathrm{A}$ and $p=\mathrm{B}$ (if the subscript $p$ in the parameters is not written explicitly then the parameters corresponding to $p=\mathrm{A}$ and $p=\mathrm{B}$ are equal).

\begin{tabular}{|l|l|l|l|l|l|}
\hline & $\begin{array}{l}\text { conductance } \\
\left(\mu \mathrm{S} / \mathrm{cm}^{2}\right)\end{array}$ & $\begin{array}{l}\text { potential } \\
(\mathrm{mV})\end{array}$ & $\begin{array}{l}\text { slope } \\
\left(\mathrm{mV}^{-1}\right)\end{array}$ & $\begin{array}{l}\text { threshold } \\
(\mathrm{mV})\end{array}$ & $\begin{array}{l}\text { time scale } \\
(\mathrm{ms})\end{array}$ \\
\hline$I_{p, \mathrm{~L}}$ & $g_{\mathrm{p}, \mathrm{L}}=0.1$ & $E_{\mathrm{p}, \mathrm{L}}=-60$ & - & - & - \\
\hline$I_{p, \mathrm{Na}}$ & $g_{\mathrm{p}, \mathrm{Na}}=3$ & $E_{\mathrm{p}, \mathrm{Na}}=50$ & $S_{\mathrm{p}, \mathrm{Na}}=0.25$ & $W_{\mathrm{p}, \mathrm{Na}}=-25$ & $\left(\tau_{\mathrm{p}, \mathrm{Na}} \approx 0\right)$ \\
\hline$I_{p, \mathrm{~K}}$ & $g_{p, \mathrm{~K}}=4$ & $E_{p, \mathrm{~K}}=-90$ & $S_{p, \mathrm{~K}}=0.25$ & $W_{p, \mathrm{~K}}=-25$ & $\tau_{p, \mathrm{~K}}=2$ \\
\hline$I_{p, \mathrm{gl}}$ & $\begin{array}{l}g_{\mathrm{A}, \mathrm{gl}}=0.196 \\
g_{\mathrm{B}, \mathrm{gl}}=0.15\end{array}$ & $E_{p, \mathrm{gl}}=50$ & $S_{p, \mathrm{gl}}=1$ & $W_{p, \mathrm{gl}}=-20$ & $\tau_{p, \mathrm{gl}}=30$ \\
\hline$I_{\mathrm{B}, \mathrm{ox}}$ & $g_{\mathrm{B}, \mathrm{ox}}=0.2$ & $E_{\mathrm{B}, \mathrm{ox}}=50$ & $S_{\mathrm{B}, \mathrm{ox}}=1$ & $W_{\mathrm{B}, \mathrm{ox}}=-20$ & $\begin{array}{l}\tau_{\mathrm{ox}}=300 \\
\tau_{\mathrm{ox}}^{+}=7500 \\
\tau_{\mathrm{ox}}^{-}=920\end{array}$ \\
\hline$I_{p, \mathrm{local}}$ & $\kappa_{p}=0.1$ & & & & \\
\hline$I_{\mathrm{ext}}$ & $I_{0}=0.893 \mathrm{~mA}$ & & & & \\
& & & & & \\
$\tau$
\end{tabular}


$(k=1)$ and sleep $(k=2)$ periods. The coefficient $r$ can vary in the interval $(-1,1)$, the maximum (minimum) value $r=1(r=-1)$ corresponding to the best (worst) possible cycle. The optimal state $r=1$ can be obtained only for $\Delta t^{(1)}=\tau_{1}$ (being awake during the whole optimal wakefulness time), and $\Delta t^{(2)}=0$ (never being awake during the optimal sleep period $\tau_{2}$ ). Any deviation implies that $\Delta t^{(1)}<\tau_{1}$ or $\Delta t^{(2)}>0$ so that $r<1$.

\section{Results}

The effect of diversity has been studied numerically by using a secondorder Runge-Kutta integration scheme, with rescaled time step $\delta t=0.01$. Heterogeneity was introduced in the values of the glutamate thresholds for a generic neuron $k$ by setting $W_{p, \mathrm{gl}} \rightarrow W_{p, \mathrm{gl}}^{k}$ and analogously for the orexin thresholds $W_{B, \text { ox }} \rightarrow W_{B, \text { ox }}^{k}$. For a given parameter $W$, different $k$ 's correspond to different values of the parameter The specific values of the diversified values used $\left\{W^{k}\right\}$ were extracted from a symmetrical bell-shaped distribution $f(W)$ centered around the value $\langle W\rangle$ of the homogeneous system in order to carry out a sensible comparison. We have used a probability density $f(W)=\mathcal{N} / \cosh ^{2}[(W-\langle W\rangle) / \delta W]$, where $\mathcal{N}$ is a normalization constant, that is convenient numerically since the corresponding cumulative distribution can be inverted exactly. The value of the diversity parameter $\delta W$ in the probability density $f(W)$ is employed below as a measure of the diversity of the system parameters and is proportional to the standard deviation, $\delta W=\pi \sigma_{W} / \sqrt{12}$, since the variance is given by $\sigma_{W}^{2}=\left\langle(W-\langle W\rangle)^{2}\right\rangle=12 \delta W^{2} / \pi^{2}$.

In this exploratory study for the sake of simplicity we have chosen $N_{\mathrm{A}}=$ $N_{\mathrm{B}}$ and a one-to-one connection between neurons of different types, i.e., each neuron $\mathrm{A}$ is coupled to one and only one neuron $\mathrm{B}$ and vice-versa. For a list of the values of the parameters used see Table 1.

In order to show more clearly the constructive effects of diversity, in the simulations we have started from a homogeneous reference state whose parameters values produce a double-periodic response to the circadian input — with period $2 \tau$. Such a response corresponds to a low-quality sleepwake cycle, since it represents absence of sleep every second night. Such an under-threshold (non-optimal) configuration with homogeneous parameters is intentionally chosen as comparison term with the other inhomogeneous systems since it best reveals the effects of added heterogeneity, namely (1) to restore single-periodicity and (2) to reduce the total sleeping time (or to 

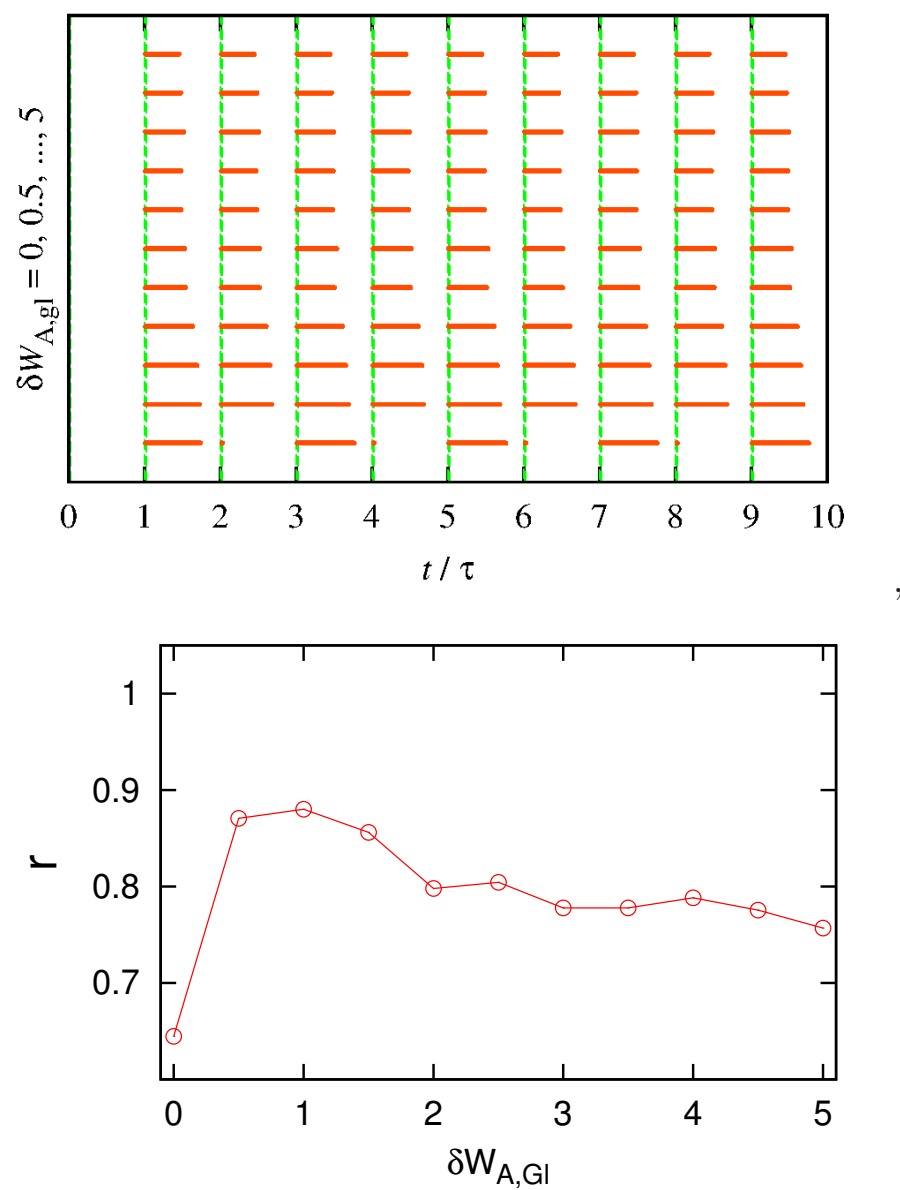

Figure 5: Raster plots (top) and corresponding $r$ coefficient defined in Eq. (15) (bottom) of a B-neuron of the set $\mathbb{B}$ when the A-neurons form a random 10-neuron network with connection probability $P=0.2$ and B-neurons are on a ring structure with first-neighbor links. In the plots above time is measured in daily periods and different ordinates (arbitrary units) correspond to different levels of diversity in the glutamate threshold of A-neurons (from bottom to top: $\delta W_{\mathrm{A}, g l}=0 \ldots 5 \mathrm{mV}$ in intervals of $0.5 \mathrm{mV}$ ).

increase it too much, see examples below) as diversity increases even more. These effects result in an optimal value of diversity at intermediate values at which the sleep-wake cycle is better.

Results for some examples of systems are presented in Figs. 5-8. All the 

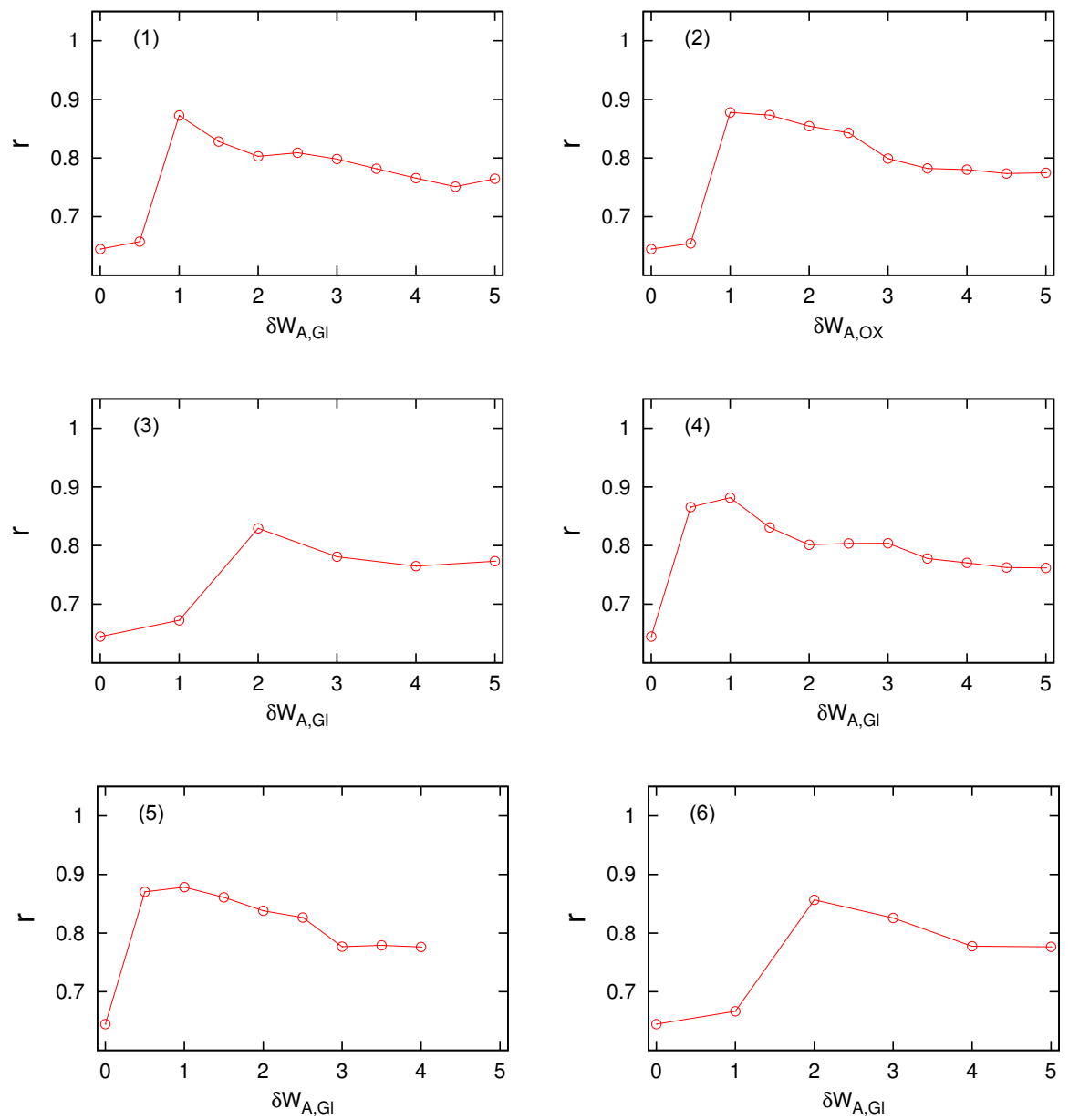

Figure 6: The $r$ coefficient obtained from Eq. (15) for some other configurations. (1): two interacting ring structures of $\mathbb{A}$ and $\mathbb{B}$ with $N_{\mathrm{A}}=N_{\mathrm{B}}=10$ neurons with first-neighbor interaction in which the glutamate threshold $W_{\mathrm{A}, g l}$ was diversified. (2): as in (1) but with diversified $W_{\text {ox }}$. (3): as in (1) but with second-neighbor interaction. (4): as in (1) but $\mathbb{A}$ is a small world network of $N_{\mathrm{A}}=10$ A-neurons with second-neighbor interaction and $P=0.25$ rewiring probability. (5): as in (4) but with $P=0.5$ (e) rewiring probability. (6): both $\mathbb{A}$ and $\mathbb{B}$ are small-world networks as $\mathbb{A}$ in (5) with $N_{\mathrm{A}}=N_{\mathrm{B}}=10$.

systems studied present a clear diversity-induced resonance, i.e. a maximum value of $r$ in correspondence of an intermediate value of diversity.

Figure 5 presents the raster plots (top) and the corresponding $r$ coefficient 

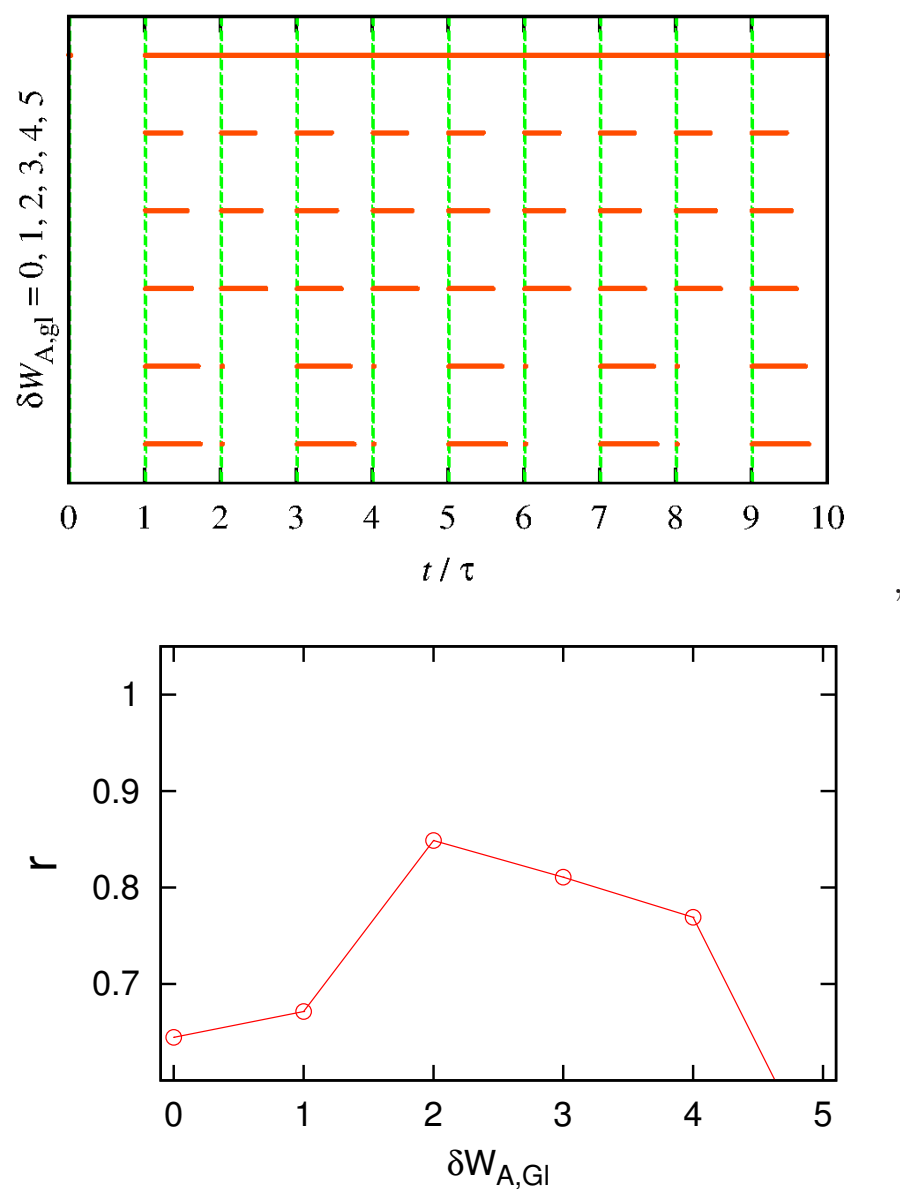

Figure 7: Same as for Fig. 5 but for to two interacting small-world networks $\mathbb{A}$ and $\mathbb{B}$ with second-neighbor interactions, $N_{\mathrm{A}}=N_{\mathrm{B}}=25$, and rewiring probability $P=0.5$.

defined in Eq. (15) of a B-neuron of the set $\mathbb{B}$ (bottom) when the A-neurons form a random 10-neuron network with connection probability $P=0.2$ and B-neurons are on a ring structure with first-neighbor links. Time is measured in daily periods. Different ordinates (arbitrary units) correspond to the different levels of diversity in the glutamate threshold of A-neurons indicated in the figure. Diversity-induced resonance results from the fact that increasing the diversity level further, after the single periodicity is restored, results in shortening more and more the sleep time. 


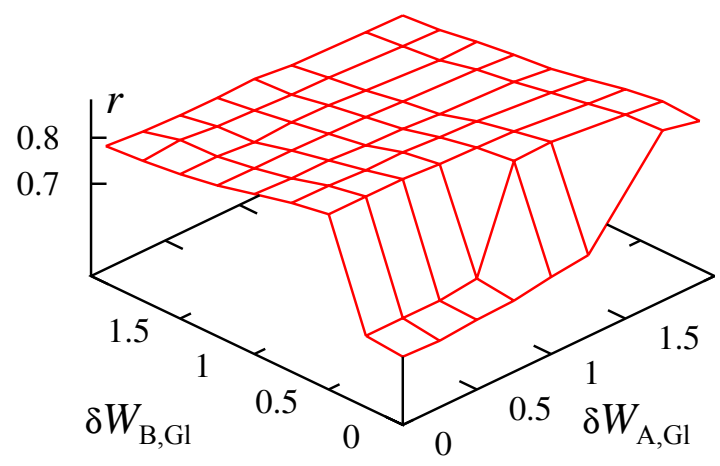

Figure 8: Coefficient $r$ for to two interacting small-world networks $\mathbb{A}$ and $\mathbb{B}$ with secondneighbor interactions, $N_{\mathrm{A}}=N_{\mathrm{B}}=10$, and rewiring probability $P=0.5$, in which both the glutamate threshold of the $\mathbb{A}$ set and that of the $\mathbb{B}$ set, $W_{\mathrm{A}, \mathrm{gl}}$ and $W_{\mathrm{B}, \mathrm{gl}}$, are diversified. The figure shows the area close to the origin of the $\delta W_{\mathrm{A}, \mathrm{gl}}-\delta W_{\mathrm{B}, \mathrm{gl}}$, where the system is homogeneous, presenting a local minimum. At large values of $\delta W_{\mathrm{A}, \mathrm{gl}}$ and $\delta W_{\mathrm{B}, \mathrm{gl}}, r$ decreases again, even if slowly.

The plots in Fig. 6 present analogous results through the raster plots obtained for different topologies of the system.

Figure 7 refers to two interacting $\mathbb{A}$ and $\mathbb{B}$ small-world networks. Notice how - on the contrary of the examples above - in this case a sharp maximum in the coefficient $r$ is due to the fact that too much diversity leads to an insomnia state with no sleep (first raster plot from top).

Finally, Fig. 8 presents the coefficient $r$ for two interacting small-world networks in which both the glutamate thresholds $\delta W_{\mathrm{A}, \mathrm{gl}}$ and $\delta W_{\mathrm{B}, \mathrm{gl}}$ have been diversified. One can see that in this case both diversified parameters contribute to improve the response of the system and that around the origin of the $\delta W_{\mathrm{A}, \mathrm{gl}}-\delta W_{\mathrm{B}, \mathrm{gl}}$ plane (where the system is homogeneous) a local minimum is present due to the double-periodicity of the cycles. At at larger values of either parameters the coefficient $r$ decreases again — even if slowly.

\section{Conclusions}

In this paper we have reported about the constructive role of diversity in the collective response of a neuronal assembly undergoing a homeostatic con- 
trol having a much longer (daily) time scale than that typical of the neuronal dynamics. The effect studied can be considered as a most typical example of global phenomenon emerging from the non-linear interaction of the units of a complex system, not only because it provides a physiological model of the complex behavior of a neuronal process but also because it is based on the presence of a suitable level of heterogeneity, a common property of complex systems that by definition can only be realized in systems composed of many interacting units.

The model studied is a natural extension of physiological models of sleepwake cycle considered in previous works and contains an assembly of heterogeneous orexin-producing neurons and glutaminergic neurons interacting with each other according to some basic network topology. It was shown that for the network configurations considered a suitable level of heterogeneity in the values of the activation thresholds of the glutamate and orexin channels can improve the quality of the wake-sleep cycle and possibly re-establish the single periodicity for some special configurations. This enlarges to neuronal systems with different underlying topologies and a long-time scale, associated

to a homeostatic mechanism, the constructive role of diversity already found and studied in many other complex systems.

\section{Acknowledgements}

This paper owes much to the experience gained working together with Svetlana Postnova and Hans A. Braun. M.P. acknowledges support from the Estonian Science Foundation Grant no. 9462 and from IUT39-1 Project. E.H-G. and R.T. acknowledge support from FEDER and MINECO (Spain) through the project INTENSE@COSYP (FIS2012-30634).

\section{References}

[1] C. Tessone, C. Mirasso, R. Toral, J. Gunton, Diversity-induced resonance, Phys. Rev. Lett. 97 (2006) 194101.

[2] C. Tessone, A. Sciré, R. Toral, P. Colet, Theory of collective firing induced by noise or diversity in excitable media., Phys Rev E 75 (2007) 016203.

[3] N. Komin, A. Murza, E. H. García, R. Toral, Synchronization and entrainment of coupled circadian oscillators, Interface Focus 1 (2010) 167176. 
[4] R. Toral, C. Tessone, Finite Size Effects in the Dynamics of Opinion Formation, Comm. Comp. Phys. 2 (2007) 177.

[5] C. Tessone, D. Zanette, R. Toral, Global firing induced by network disorder in ensembles of active rotators, Eur. Phys. J. B 62 (2008) 319.

[6] C. Tessone, R. Toral, Diversity-induced resonance in a model for opinion formation, Eur. Phys. J. B 71 (2009) 549.

[7] T. Vaz Martins, R. Toral, M. Santos, Divide and conquer: resonance induced by competitive interactions, Eur. Phys. J. B 67 (2009) 329-336.

[8] T. Perez, C. Mirasso, R. Toral, J. Gunton, The constructive role of diversity on the global response of coupled neuron systems, Phil. Trans. Roy. Soc. A 368 (2010) 5619-5632.

[9] S. Postnova, K. Voigt, H. A. Braun, A mathematical model of homeostatic regulation of sleep-wake cycles by Hypocretin/Orexin, Journal of Biological Rhythms 24 (2009) 523-535.

[10] C. Peyron, D. K. Tighe, A. N. van den Pol, L. de Lecea, H. C. Heller, J. G. Sutcliffe, T. S. Kilduff, Neurons containing hypocretin (orexin) project to multiple neuronal systems., J Neurosci 18 (23) (1998) 999610015 .

[11] K. Yoshida, S. McCormack, R. A. España, A. Crocker, T. E. Scammell, Afferents to the orexin neurons of the rat brain., J Comp Neurol 494 (5) (2006) 845-61.

[12] R. Winsky-Sommerer, A. Yamanaka, S. Diano, E. Borok, A. J. Roberts, T. Sakurai, T. S. Kilduff, T. L. Horvath, L. de Lecea, Interaction between the Corticotropin-Releasing Factor System and Hypocretins (Orexins): A Novel Circuit Mediating Stress Response, The Journal of Neuroscience 24 (2004) 11439.

[13] T. Sakurai, The neural circuit of orexin (hypocretin): maintaining sleep and wakefulness., Nature Rev. Neurosci. 8 (3) (2007) 171-81.

[14] Li Y., Gao X.B., Sakurai T., van den Pol A.N., Hypocretin/orexin excites hypocretin neurons via a local glutamate neuron-A potential mechanism for orchestrating the hypothalamic arousal system, Neuron 36 (2002) 1169-1181. 
[15] A. B. et al., A two process model of sleep regulation, Hum. Neurobiol. 1 (1982) 195-204.

[16] M. Patriarca, S. Postnova, H. Braun, E. Hernández-García, R. Toral, Diversity and Noise Effects in a Model of Homeostatic Regulation of the Sleep-Wake Cycle, PLoS Comput. Biol. 8 (8) (2012) e1002650.

[17] M. Patriarca, S. Postnova, H. Braun, E. Hernández-García, R. Toral, Modelling the homeostatic regulation of the sleep-wake cycle: role of diversity, Presented at NOLTA 2012 - International Symposium on Nonlinear Theory and its Applications, Palma de Mallorca, Spain, 2012 October 22nd-26th.

[18] Eggermann E., Bayer L., Serafin M., The wake-promoting hypocretinorexin neurons are in an intrinsic state of membrane depolarization, J. Neurosci. 23 (2003) 1557-62. 